\title{
Immediate Ultrasound Calibration with Three Poses and Minimal Image Processing
}

\author{
Anand Viswanathan, Emad M. Boctor, \\ Russell H. Taylor, Gregory Hager, and Gabor Fichtinger \\ Engineering Research Center, Johns Hopkins University \\ \{anand, rht, hager, gabor\}@cs.jhu.edu, eboctor@ieee.org
}

\begin{abstract}
This paper introduces a novel method for ultrasound (US) probe calibration based on closed-form formulation and using minimal US imaging allowing for an immediate result. Prior to calibration, a position sensor is used to track each image in 3D space while the US image is used to determine target location within the image. The calibration procedure uses these two pieces of information to determine the transformation (translation, rotation, and scaling) of the scan plane with respect to the position sensor. We utilize a closed form solution from two motions of the US probe relying on optical digitization with a calibrated pointer to replace with a great extent the traditional segmentation of points/planes in US images. The tracked pointer appeared to introduce significantly less error than the resolution of the US image caused in earlier approaches. Our method also uses significantly fewer US images and requires only minimal image segmentation, or none with a special probe attachment.
\end{abstract}

\section{Introduction}

Ultrasound imaging (US) has emerged as a widely popular guidance modality for medical interventions, since it is real-time, safe, convenient to use in the operating room, and inexpensive compared to other modalities such as CT and MRI. Significant research has been dedicated to using US in a quantitative manner. Toward this goal, the main problems are to assemble individual 2D US images into 3D volumes [1] and then to relate the position of surgical tools with respect to the reconstructed US volume. Solution of both problems requires tracking the 2D US probe in 3D space with respect to a stationary frame of reference. Typically, tracking is achieved by rigidly attaching $3 \mathrm{D}$ localizers to the US probe. The missing link, however, is the spatial transformation between the US image pixels and the tracking body attached to the US probe, which requires calibration. Hence, calibration is ubiquitously present in all systems where 2D ultrasound is used for quantitative image guidance. Clearly, the accuracy of calibration is the most significant factor in the accuracy of these systems.

In all currently known calibration processes, an object of known geometrical properties (a.k.a. phantom) is scanned by the tracked US probe and then various mathematical procedures are applied to determine the unknown transformation that maximizes the similarity between the US images and the actual phantom. Geometrical phantoms based on points $[2,3,4,5,6]$ and planes $[7,4,6]$ have been developed and 
compared in terms of accuracy and performance $[4,6]$. The cross-wire and threewire phantoms require large numbers of images and are hard to automate, while the single-wall phantom as in Cambridge phantom [4] is a more automatic, repeatable method. Typically however, all of these phantoms are very complex to create and to use in calibration. These complex phantoms also require segmentation of numerous points per each individual US image. Figure 1 shows a typical formulation for the coordinate systems required for the previously mentioned phantoms.

There is error associated with each stage of the calibration process (typically phantom fabrication, image acquisition, spatial co-registration, image processing, formulation of transformations, and numerical optimization solution) which aggregate and may induce a prohibitively large final error in the calibration.

\section{Mathematical Formulation}

In our new framework, we still image a calibration phantom submerged in water bath. We will demonstrate, however, that in comparison to standard calibration phantoms, the complexity of the phantom is minimal and the number of images required for calibration is remarkably low (3-6 frames). Figure 2 presents the coordinate systems for mathematical formulation. $\mathrm{A}_{1}, \mathrm{~A}_{2}$ are the transformations of US image coordinate system $(P)$ with respect to the fixed reconstruction coordinate system $(C)$ at poses 1 and 2, respectively. The actual selection of $\mathrm{C}$ is arbitrary and the only requirement is that it must be rigidly fixed during the calibration process. Using $\mathrm{A}_{1}, \mathrm{~A}_{2}$, we obtain the transformation between

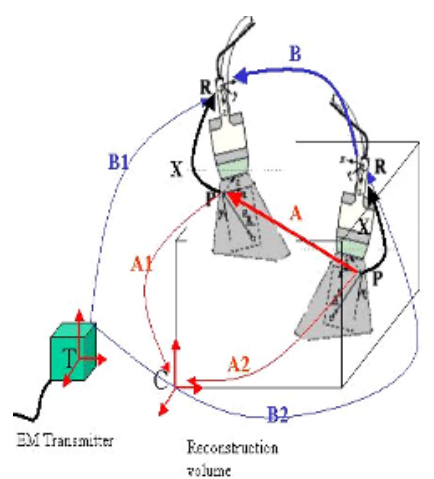

Fig. 2. The coordinate systems formulation for the proposed $\mathrm{AX}=\mathrm{XB}$ method. poses 1 and 2, as $A=A_{2} A_{1}^{-1}$. At the same time, the transformation between the two poses can be recovered using a calibration phantom or recovered directly by matching 
the $2 \mathrm{D}$ ultrasound images acquired in these poses to a prior $3 \mathrm{D}$ model of the phantom object. To determine the matrix A, we focused on a strategy that minimized the complexity of our imaging phantom. $\mathrm{A}_{1}, \mathrm{~A}_{2}$ are the relative transformations between each of our imaging phantoms. $\mathrm{B}_{1}, \mathrm{~B}_{2}$ are the tracking device readings for the sensor frame $(R)$ with respect to tracker reference frame $(T)$ at poses 1 and 2 respectively. Again, the relative pose between sensor frame $(R)$ at pose 1 and 2 is given by $B=B_{2}^{-1} B_{1}$. This yields the following homogeneous matrix equation:

$$
\mathrm{AX}=\mathrm{XB}
$$

Where A is estimated from images, B is assumed to be known from the external tracking device, and $\mathrm{X}$ is the unknown transformation between the US image coordinate system and the sensor frame $(R)$. The estimated US image frame motion in general is given by:

$$
A(\lambda)=\left(\begin{array}{cccc} 
& & \lambda_{x} \cdot u_{a x} \\
& R_{a} & & \lambda_{y} \cdot u_{a y} \\
& & & \lambda_{z} \cdot u_{a z} \\
0 & 0 & 0 & 1
\end{array}\right)
$$

Where $R_{a}$ is the rotation of the US image frame between pose 1 and $2, \lambda$ is the unknown scale factor vector that relates the translation vector $\mathrm{u}_{\mathrm{a}}$ in voxel space (3DUS, $\mathrm{CT}$, or MRI) to the US image frame translation vector $\mathrm{t}_{\mathrm{a}}$ (in $\mathrm{mm}$ ) such that

$$
t_{a}=\left(\begin{array}{c}
\lambda_{x} u_{a x} \\
\lambda_{y} u_{a y} \\
\lambda_{z} u_{a z}
\end{array}\right)=\left(\begin{array}{ccc}
u_{a x} & 0 & 0 \\
0 & u_{a y} & 0 \\
0 & 0 & u_{a z}
\end{array}\right) \cdot\left(\begin{array}{c}
\lambda_{x} \\
\lambda_{y} \\
\lambda_{z}
\end{array}\right)=D_{u a} \lambda .
$$

It is important to account for the most general case where the scale factor $\lambda$, which converts from voxel space to metric coordinates, is not known. Such a scenario could happen if " $A$ " is recovered by registering the US image to a prior acquired model in voxel space. From the homogeneous equation (1) and using (2), one obtains:

$$
\begin{gathered}
R_{a} R_{x}=R_{x} R_{b} \\
R_{a} t_{x}+D_{u a} \lambda=R_{x} t_{b}+t_{x}
\end{gathered}
$$

In the linear formulation of the problem we will use the linear operator vec and the Kronecker product $(\otimes)[10]$. Using the following fundamental property of the Kronecker product:

$$
\operatorname{vec}(C D E)=\left(C \otimes E^{T}\right) \operatorname{vec}(D)
$$

One can rewrite (4) and (5) into:

$$
\left(R_{a} \otimes R_{b}\right) \operatorname{vec}\left(R_{x}\right)=\operatorname{vec}\left(R_{x}\right), \text { and }
$$




$$
\left(I_{3} \otimes t_{b}^{t}\right) \operatorname{vec}\left(R_{x}\right)+\left(I_{3}-R_{a}\right) t_{x}-D_{u} \lambda=0
$$

From (7) and (8), we can transform the whole problem ( $\mathrm{XX}=\mathrm{XB})$ into a single homogeneous linear system:

$$
\left[\begin{array}{ccc}
I_{9}-R_{a} \otimes R_{b} & 0_{9 * 3} & 0_{9 * 3} \\
I_{3} \otimes t_{b}^{t} & I_{3}-R_{a} & -D_{u}
\end{array}\right]\left(\begin{array}{c}
\operatorname{vec}\left(R_{x}\right) \\
t_{x} \\
\lambda_{3 * 1}
\end{array}\right)=\left(\begin{array}{c}
0_{9 * 1} \\
0_{3 * 1}
\end{array}\right)
$$

The solution for this homogeneous linear system could be given by finding the null space, which is a subspace in $R^{15}$. Then the unique solution could be extracted from the null space using the unity constraint to the first 9 coefficients representing the $R_{x}$. However, a better solution is described in [9] where the system is solved in two steps: first extract the rotation, and then solve for the translation and scale. The complete algebraic analysis for this problem (where the scale factor is assumed to be constant in three direction) is given in [9], where it is proved that two independent motions with non-parallel axes is sufficient to recover a unique solution for $\mathrm{AX}=\mathrm{XB}$. We have extended this solution method to account for inhomogeneous scale in the three coordinate axes.

\section{Calibration Setup and Protocol}

We have introduced the above closed form formulation before using a modified Zshape phantom [8], which is tedious to build and process it. In this paper we have replaced the z-phantom with an easier design to build and to process as shown in Figure 3. In our experimental setup, we used the SONOLINE Antares US scanner (Siemens Medical Solutions USA, Inc. Ultrasound Division, Issaquah, WA) with a Siemens VF 10-5 linear array probe held in a rigid attachment mounted on an adjustable arm. The
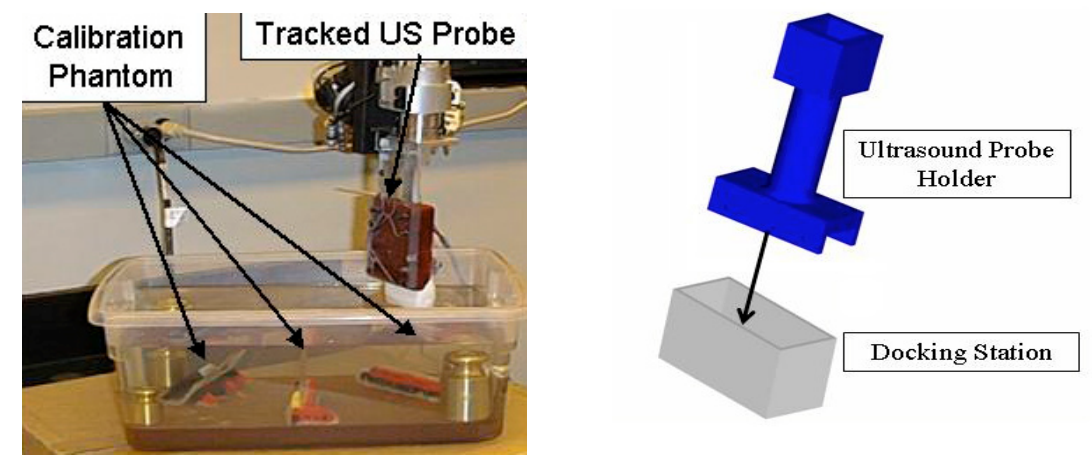

Fig. 3. Calibration setup: Optically tracked probe, which images the calibration phantom (L). A sketch of the planned "docking station" for ultrasound probe calibration. 
adjustable arm is used to adjust the spatial position of the tracked US probe to image the calibration phantom. Multiple optical markers were attached to the probe holder, which then were tracked by an OPTOTRAK device (Northern Digital Inc.). The calibration phantom was submerged in a transparent plastic water tank. The calibration phantom consisted of three identical thin $(4 \mathrm{~mm})$ plastic plates of irregular shape. The candidate feature from this phantom is simply a single point on a line that can be easily detected by a wide variety of probes and depth settings. The plastic plates were machined together to ensure their congruency. The plates were positioned on a flat surface and fixed in place using Lego blocks and permanent glue for support, as seen in Figure 4. Using an optical pointer, we collected 3D points of each of the plates for offline processing. The pointer was pivoted about each digitized point in order to obtain an accurate estimate of the desired 3D point as shown in Figure 4. These 3D points are registered to provide a local coordinate system for each of the thin plates. From the local coordinate systems, one can calculate the relative transformations between each pair of plates. The poses of the three plates were carefully arranged in order to give the optimal results for the two motions required by the $\mathrm{AX}=\mathrm{XB}$ formulation, based on previous experiments.

Traditionally in US probe calibration research; arrays of wires have been used to establish the relationship between the coordinate systems of the US and the tracking device. This approach typically involves laborious segmentation to extract the wire points in the US images and then relate these points to the tracking device coordinate system. In this paper, we take a different approach by computing the $A_{1}$ and $A_{2}$ first by locating prominent feature points of the plates in the US images and then using an optical digitizer (Figure 4) to compute the relative transformation between the plates. We tested two protocols for computing the $\mathrm{A}_{1}$ and $\mathrm{A}_{2}$ matrices for our thin plate calibration phantom:
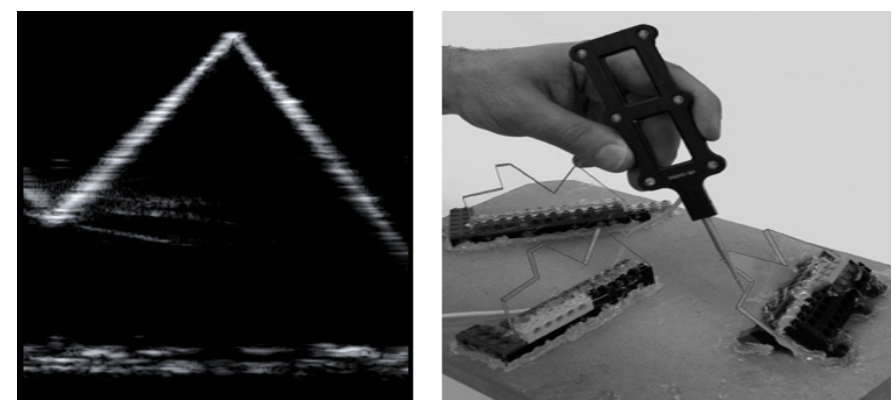

Fig. 4. Optical pointer is used to digitize $3 \mathrm{D}$ points prior to calibration on the three clear plastic plates forming the phantom (R), and an ultrasound image of one of the plates (L).

1. Move the US tracked probe such that the probe is parallel to the thin plate and the image plane of the US image shows the middle of the thin plate.

2. Apply the same protocol as above, but collect multiple tracking data from one end of the thin plate to the other end and use an averaging technique to find the "center" of the thin plate.

By using the adjustable arm, we receive consistent and good appearance of the thin plates in the US images. As a practical alternative, we are currently developing a sim- 
ple probe holder that will dock into the thin plates in a predefined manner, thereby guaranteeing correct alignment between the probe and the plates. This concept can be seen in Figure 3. The same concept (i.e. using a mechanical attachment to the US probe) has been successful in the Cambridge phantom [4]. The consistent placement of the probe on the plates yields predictable and sharp US images that could even be processed automatically. Automatic image processing, however, might not promise a significant advantage, as we only use 3-6 frames and just a single feature point and a line in each.

In Figure 4, An US image of a portion of the calibration phantom is shown. To acquire this image, the probe is placed according to the protocol to make sure that the entire plane of the plate appears in the US image at once. To ensure in-plane alignment of the US probe and a plate being scanned, the contour of the plate is observed as the probe is moved over the plate. The probe and plate being in the same plane forms a constraint from which we assume that the $A_{1}$ and $A_{2}$ transformation matrices are the relative transformations between the plates of the calibration phantom with a positional offset based on the pixel coordinates of the phantom in the acquired US image. From the US image, we observe the sharp contours of the plates (Figure 4) from which we compute the rotation of the plate within the plane of the US.

Three sets of tracking and US image data are sufficient to solve the mathematical formulation with acceptable accuracy. An additional 3 sets of data can generate 48 calibration datasets, which will ensure a well-conditioned problem and produce comparable results to previous calibration accuracies [4]. Furthermore, when the probe is held in a docking attachment, the US images need to be collected and processed only once during the lifetime of the probe and any future calibration can be performed without relying on any US images.

\section{Experiments and Results}

To test the numerical stability of the closed form formulation, simulation data was generated. Artificial noise was added to the data points to mimic the error of the tracker and account for the effects of ultrasound image properties. Accordingly, the following protocol aims to simulate these disturbances. First, the missing transformation $\mathrm{X}$ is picked by a random choice. Second, a sequence of probe motions is chosen. From the unknown transformation $\mathrm{X}$, the ultrasound image motion can be deduced.

Table 1. Average error and standard deviation of the recovered translation vector for different calibration sequences. The sequences were generated using synthetic data with added noise of $.5 \%, 1 \%, 5 \%, 10 \%$ respectively.

\begin{tabular}{|c|ccc|ccc|}
\cline { 2 - 7 } Noise Level (\%) & \multicolumn{4}{|c|}{ Average Error $(\mathrm{mm})$} & \multicolumn{3}{c|}{ Standard Dev. $(\mathrm{mm})$} \\
\hline 0.5 & 0.0013 & -0.00113 & -0.0668 & 0.468 & 0.125 & 0.298 \\
\hline 1 & -0.002 & 0.00652 & 0.0356 & 0.382 & 0.195 & -0.109 \\
\hline 5 & 0.0657 & -0.0357 & -0.888 & 8.726 & 2.132 & 4.512 \\
\hline 10 & 0.0461 & -0.0160 & -0.895 & 13.89 & 3.459 & 6.058 \\
\hline
\end{tabular}


Table 2. Average pose and deviation of the recovered calibration matrix using the thin plate calibration phantom with the $\mathrm{AX}=\mathrm{XB}$ method.

\begin{tabular}{|c|rrr|rrr|}
\cline { 2 - 6 } \multicolumn{1}{c|}{} & \multicolumn{3}{c|}{ Average } & \multicolumn{3}{c|}{ Standard Dev.(repeatability) } \\
\hline Position (mm) & 93.83 & -38.50 & 38.21 & 1.31 & 2.08 & 2.41 \\
\hline $\begin{array}{c}\text { Roll, Pitch, and Yaw } \\
\text { Angles (degrees) }\end{array}$ & 177.85 & 0.29709 & 2.7751 & \multicolumn{3}{c}{0.74} \\
\hline
\end{tabular}

Third, different levels of white noise are added to the ultrasound image motion frame "A's" to simulate a real environment as well as to the simulated tracking readings "B's". The resolution of a tracking device is always in the order of $0.1 \%$ for EM devices and less than $0.001 \%$ for an optical based system. The algorithm was executed to recalculate the $\mathrm{X}$ transformation under different noise conditions (Table 1.)

In order to check the repeatability of our calibration setup and our phantom's performance, we gathered data using a tracked ultrasound probe. Real US data was acquired in 2 poses for each of the calibration plates. The algorithm was tested on 48 unique combinations of 6 different poses (two poses per plate). Table 2 reports the average recovered pose values of these 48 combinations as well as the standard deviation, which reflect the repeatability of this method. These numbers are comparable to the previously reported repeatability analysis [4]. Right after the data collection (tracking information and the offline processed data), the calibration algorithm executes almost immediately. The major source of expected error stems from the misalignment of the ultrasound probe to the plane of the thin plate. With the addition of the planned docking station, this source of error can be removed from the system.
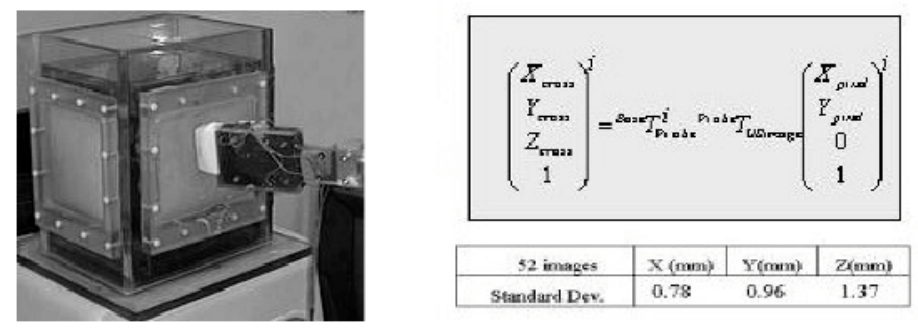

Fig. 5. Reconstruction accuracy setup, acquiring 52 cross wire images from different angles (L), The reconstruction precision framework ( $\mathrm{R}$, Top) and the resultant standard deviation in $\mathrm{mm}$ (R, Bottom).

Next we performed precision assessment for the calibration, using one of the reported accuracy analysis methods $[4,5]$. Reconstruction precision based on 3D reconstruction of the cross wire. The basic idea behind this test is to check how precise the calibration matrix would reconstruct all the cross wire points gathered from different insonification angles into a fixed point in space. The standard deviation of the point cloud reflects the uncertainty in the calibration matrix as well as the manual extraction of the cross wire points, as shown in Figure 5. The resulting calibration precision is highly comparable to [4]. 


\section{Discussion}

These preliminary results indicate significant potential in using a simple calibration phantom in conjunction with the $\mathrm{AX}=\mathrm{XB}$ closed form formulation. The sharp features of thin plastic plates of the calibration phantom appear markedly in US images and it is easy to locate these features based on pixel intensity and gradient. Compared to conventional wire-based calibration phantoms, the simplified design of our phantom and the use of optical digitization of 3D feature points reduced the amount of image processing required and still provided very accurate calibration results. Because conventional calibration methods relied on image information from an US machine, registration accuracy, to a large extent, also depended on the resolution of the US imaging system and the accuracy of feature extraction. Ultrasound noise and beam width problem would normally affect the accuracy of the estimated A matrix. Now, by relying on the OPTOTRAK, which has $0.02 \mathrm{~mm}$ resolution and $0.5 \mathrm{~mm}$ accuracy, we achieved a significantly more reliable calibration. Based on the simulation experiments, the closed formed formulation still produces stable calibration matrix. Therefore if the docking station was used to eliminate the misalignment error between the US probe and the phantom, we should expect sub millimeter reconstruction precision. The simple docking guide mechanism eliminates the need for the currently used adjustable arm and allow for offline image processing leading to immediate calibration.

Acknowledgements. This work is supported by NSF EEC 9731478. We are grateful to Carol Lowery, Levin Nock, and David Gustafson (Siemens Medical Solutions USA, Inc. Ultrasound Division, Issaquah, WA) for providing Ultrasound Antares unit equipped with URI interface.

\section{References}

1. A. Fenster, D. B. Downey, and N. H. Cardinal, "Three Dimensional Ultrasound Imaging," Physics in medicine and biology, vol 46, pp. 67-99, 2001.

2. P. R. Detmer, G. Bashein, T. Hodges, K. W. Beach, E.P. Filer, D. H. Burns, and D. E. Strandness Jr., "3D Ultrasonic Image Feature Localization based on Magnetic Scanhead Tracking: In Vitro Calibration and Validation." US in Med. Biol., 23(4):597-609, 1996.

3. J. Carr, Surface Reconstruction in 3D Medical Imaging, Ph.D. thesis, University of Canterbury, Christchurch, New Zealand, 1996.

4. R. W. Prager, Rohling R. N., Gee A. H., and Berman L., "Rapid Calibration for 3-D Freehand Ultrasound," US in Med. Biol., 24(6):855-869, 1998.

5. N. Pagoulatos, D. R. Haynor, and Y. Kim, "A Fast Calibration Method for 3-D Tracking of Ultrasound Images Using a Spatial Localizer," US in Med. Biol., 27(9):1219-1229, 2001.

6. Emad M. Boctor, A. Jain, M. Choti, Russell H.Taylor, Gabor Fichtinger, "Rapid calibration method for registration and 3D tracking of ultrasound images using spatial localizer," Proc. SPIE Medical Imaging, Vol. 5035,p. 521-532, 2003.

7. F. Rousseau, P. Hellier, C. Barillot, "A fully automatic calibration procedure for freehand 3D ultrasound," In IEEE Int. Symp. on Biomedical Imaging, Washington D.C, Juillet 2002. 
8. Emad Boctor, Anand Viswanathan, Michael Choti, Russell H. Taylor, Gabor Fichtinger, and Gregory Hager, "A Novel Closed Form Solution for Ultrasound Calibration," In IEEE Int Symp. On Biomedical Imaging, 2004.

9. Nicolas Andreff and Radu Horaud and Bernard Espiau, "Robot Hand-Eye Calibration Using Structure from Motion," International J. of Robotics Research, 20(3), pp 228-248, 2001.

10. John W. Brewer, "Kronecker Products and Matrix Calculus in System Theory", IEEE Trans. Circuits and systems, 25(9) Sep.1978. 